\title{
Experimental Investigation on Cavitating Flow Induced Vibration Characteristics of a Low Specific Speed Centrifugal Pump
}

\author{
Bo Gao, Pengming Guo, Ning Zhang, Zhong Li, and Minguan Yang \\ School of Energy and Power Engineering, Jiangsu University, Zhenjiang 212013, China \\ Correspondence should be addressed to Ning Zhang; nzhang@ujs.edu.cn
}

Received 20 March 2017; Accepted 20 June 2017; Published 31 July 2017

Academic Editor: Tony Murmu

Copyright (c) 2017 Bo Gao et al. This is an open access article distributed under the Creative Commons Attribution License, which permits unrestricted use, distribution, and reproduction in any medium, provided the original work is properly cited.

Cavitating flow developing in the blade channels is detrimental to the stable operation of centrifugal pumps, so it is essential to detect cavitation and avoid the unexpected results. The present paper concentrates on cavitation induced vibration characteristics, and special attention is laid on vibration energy in low frequency band, 10-500 Hz. The correlation between cavitating evolution and the corresponding vibration energy in $10-500 \mathrm{~Hz}$ frequency band is discussed through visualization analysis. Results show that the varying trend of vibration energy in low frequency band is unique compared with the high frequency band. With cavitation number decreasing, vibration energy reaches a local maximum at a cavitation number much larger than the 3\% head drop point; after that it decreases. The varying trend is closely associated with the corresponding cavitation status. With cavitation number decreasing, cavitation could be divided into four stages. The decreasing of vibration energy, in particular cavitation number range, is caused by the partial compressible cavitation structure. From cavitation induced vibration characteristics, vibration energy rises much earlier than the usual 3\% head drop criterion, and it indicates that cavitation could be detected in advance and effectively by means of cavitation induced vibration characteristics.

\section{Introduction}

Cavitation, one of the unexpected flow instabilities inside centrifugal pumps, is detrimental to the stable operation of centrifugal pumps [1-3]. With absolute static pressure at the impeller suction decreasing, cavitation may easily occur at the blade leading edge. Furthermore, with static pressure decreasing continuously, cavitation region inside blade channels expands towards the blade trailing edge, and cavitation bubbles travel with the main flow collapsing at high static pressure region [4]. During the collapsing process, unsteady impact forces acting on the blade surfaces and shock waves would be generated resulting in vibration energy of the pump increasing rapidly $[5,6]$. Besides, due to the continuously impingement effect of the burst cavitation bubbles, cavitation erosion would develop on the blade surfaces. So it is essential to detect cavitation during pump operating to avoid the unexpected effects.

To detect cavitation inside the centrifugal pump, determination of $3 \%$ head drop is the most common method in industrial application $[7,8]$. But many researches have proved that cavitation develops much earlier than that reflected in the head curve $[9,10]$. Visualization of cavitation inside the blade channels could be applied to observe cavitation inception effectively, but it is not a convenient means in industrial application [11]. Along with cavitation occurring, emitted noise and high amplitude vibration would be easily identified [12, 13]. So cavitation induced vibration signals could be applied to detect cavitation considering its convenient characteristics for application.

Cudina $[14,15]$ applied acoustic method to detect cavitation developing in the pumps and found that cavitation has a significant influence on the emitted noise of the pump. The relationship between discrete components and cavitation was discussed and carried out. Many researches have been done to investigate vibration induced by cavitation in pumps, but most of them lay emphasis upon high frequency vibration signals. Mcnulty and Pearsall [16] investigated cavitation induced noise characteristics, but vibration signals lower 


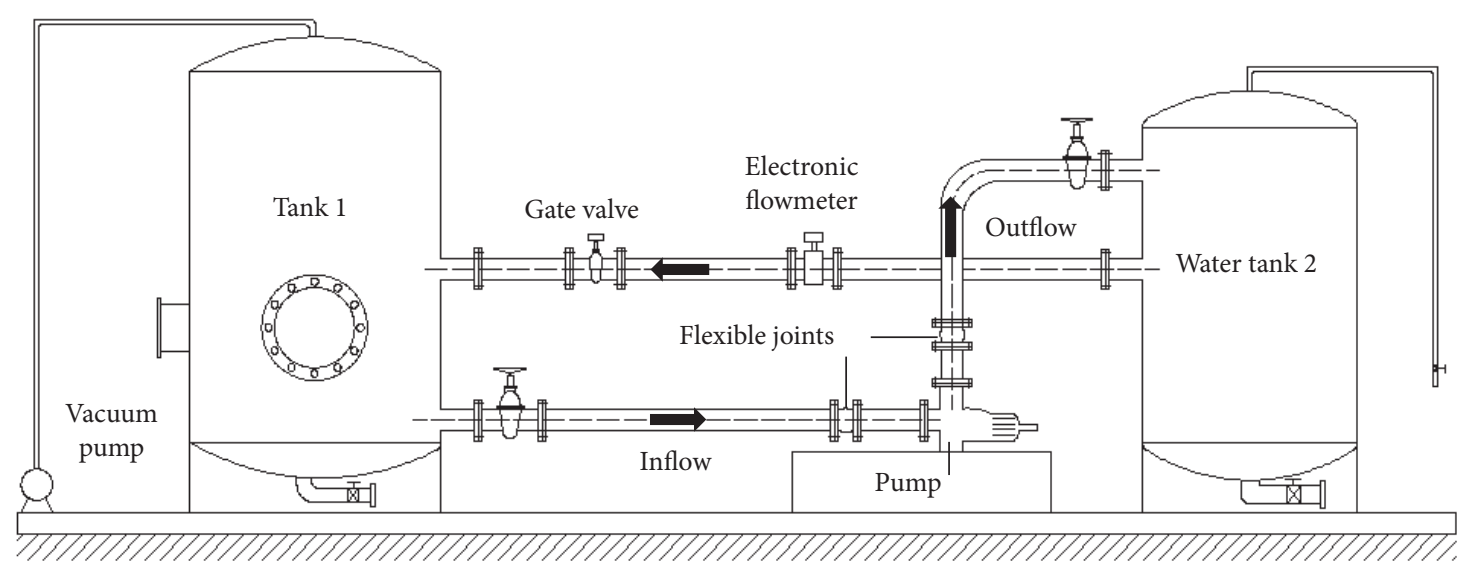

FIGURE 1: Closed test platform.

than $20 \mathrm{kHz}$ were neglected during research. Rus et al. [17] also concentrated on high signals caused by cavitation of a Kaplan turbine. Consequently, low frequency signals affected by cavitation were rarely investigated. Besides, a synthetic analysis of cavitation visualization and cavitation induced vibration is rarely conducted leading to a lack of comprehensive understanding of the influence of cavitation evolution on vibration characteristics, especially for low frequency signals.

So in the present study, visualization of cavitating flow and the corresponding induced vibration characteristics are combined to investigate the influence of cavitating flow on vibration energy of the model pump. Emphasis is laid upon the low frequency signals. The present paper attempts to illustrate the influence of cavitation on the entire frequency band signals. Besides, the correlation between cavitation evolution and vibration energy characteristics would also be clarified. Finally, a comprehensive understanding of cavitation induced vibration would be carried out to establish an effective way to detect cavitation in centrifugal pumps.

\section{Experimental Setup}

2.1. Vibration Measuring Method. A low specific speed $n_{s}=$ 69 centrifugal pump is designed for investigation, and the main parameters of the model pump are shown in Table 1. To measure vibration characteristics induced by cavitating flow, a model pump made of stainless steel is designed; besides a transparent model pump of Plexiglass is also made to visualize the cavitating flow versus cavitation number decreasing.

Experiments of cavitating flow induced vibration are conducted in a closed test loop as presented in Figure 1. By lowering the static pressure in water tank 1 using the vacuum pump, the net positive suction head at the pump inlet would be reduced gradually. Thus cavitation would easily develop on the blade suction side. The gate valve locating near the water tank 1 is applied to adjust the capacity of the model pump. The flow rate of the model pump is measured by the electronic flowmeter, which has an uncertainty of $\pm 0.2 \%$ of the measured value. The head of the model pump is obtained
TABLE 1: Main design parameters of the model pump.

\begin{tabular}{lc}
\hline Parameters & Value \\
\hline Nominal flow rate $Q_{d}$ & $55 \mathrm{~m}^{3} / \mathrm{h}$ \\
Design head $H_{d}$ & $20 \mathrm{~m}$ \\
Nominal rotating speed $n_{d}$ & $1450 \mathrm{r} / \mathrm{min}$ \\
Specific speed $n_{s}=3.65 n_{d} \sqrt{Q_{d}} / H_{d}{ }^{0.75}$ & 69 \\
Blade number $Z$ & 6 \\
Impeller inlet diameter $D_{1}$ & $80 \mathrm{~mm}$ \\
Impeller outlet diameter $D_{2}$ & $260 \mathrm{~mm}$ \\
Impeller outlet width $b_{2}$ & $17 \mathrm{~mm}$ \\
Volute inlet diameter $D_{3}$ & $290 \mathrm{~mm}$ \\
Volute outlet diameter $D_{4}$ & $80 \mathrm{~mm}$ \\
Blade outlet angle $\beta_{2}$ & $30^{\circ}$ \\
Wrap angle $\phi$ & $115^{\circ}$ \\
Tangential velocity at impeller exit $u_{2}$ & $19.7 \mathrm{~m} / \mathrm{s}$ \\
Angle of volute tongue $\alpha$ & $20^{\circ}$ \\
\hline
\end{tabular}

by using the gauge pressure with an accuracy of $\pm 0.1 \%$ of the measured value. The disturbance vibration signals from the pipes would have a significant influence on the vibration signals of the model pump. Two flexible joints are mounted at the pump inlet and outlet suctions to alleviate the interference effects from the piping systems.

To have a comprehensive understanding of vibration characteristics induced by cavitating flow, three triaxial accelerometers (PCB356A16) are mounted on the surface of the spiral volute casing as seen in Figure 2. The PCB series accelerometers used in the experiment have a flat frequency response from $0.5 \mathrm{~Hz}$ to $5 \mathrm{kHz}$ with a resonant frequency higher than $25 \mathrm{kHz}$. The relative position of the sensors with respect to the volute and the measuring direction of each sensor are shown in Table 2.

To obtain the vibration signals of the model pump, LMS measuring systems are applied, which support dynamic signal sampling by using 24 sampling channels. Besides, the maximum sampling frequency of each channel could almost 
TABLE 2: Positions and measuring directions of the accelerometers.

\begin{tabular}{lcc}
\hline Accelerometer & Position & Measuring direction \\
\hline$V 1$ & Near the volute tongue & $x, y, z$ \\
$V 2$ & The second cross-section of volute & $x, y, z$ \\
$V 3$ & The fourth cross-section of volute & $x, y, z$ \\
\hline
\end{tabular}

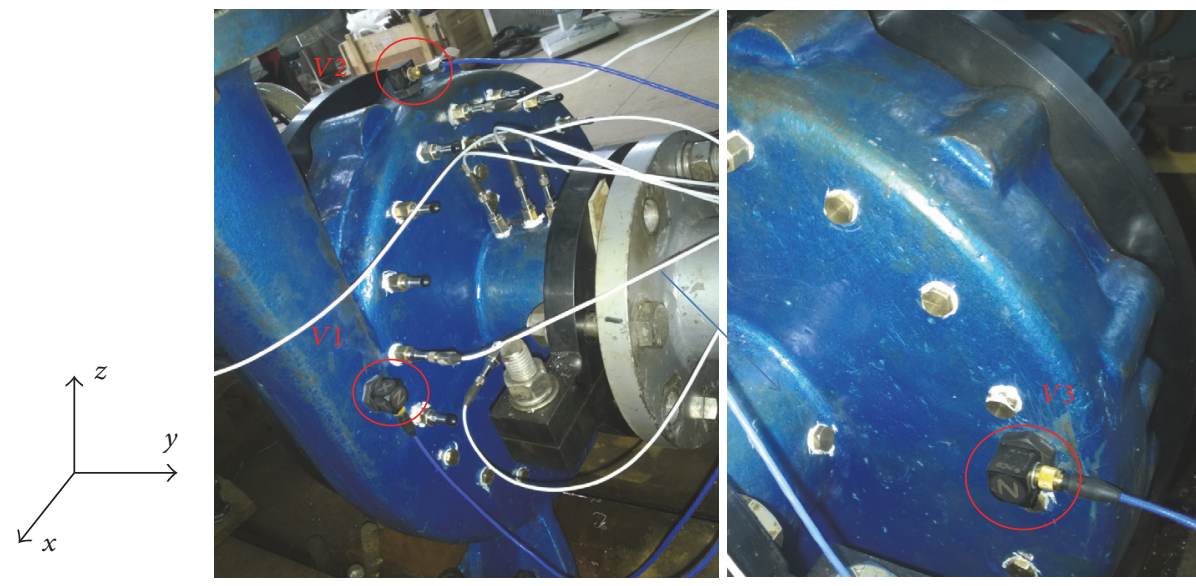

FIGURE 2: Positions of the accelerometers mounted on the volute casing.

reach $102.4 \mathrm{kHz}$, and it is adequate to acquire the vibration signals during experiment. It is accepted that the collapse time of cavitation bubble is usual on the order of magnitude of millisecond. Thus, high frequency signals would be emitted during cavitation bubbles collapsing. So to capture cavitation induced high frequency signal, the bandwidth of the vibration spectrum is set as $51200 \mathrm{~Hz}$ during signal measuring process, and it is the upper limiting frequency for the presently used accelerometers. The corresponding sampling frequency is $102400 \mathrm{~Hz}$ to satisfy Nyquist sampling theorem [18]. To reduce energy leakage of the obtained signals, Hanning window is applied during signal processing [19]. The resolution of pressure spectrum is defined as $1 \mathrm{~Hz}$. Finally, the sampling number of data used for analysis is 102400. The autopower spectrum algorithm is employed to analyze the vibration spectrum characteristics of the model pump.

2.2. Visualization of Cavitating Flow. A transparent model pump made of Plexiglass is investigated to visualize cavitating flow inside the blade channels. Figure 3 shows the transparent impeller. To capture the complex cavitating flow, high speed camera Olympus I-Speed 3 is used. The exposure time is set as $1.33 \times 10^{-4} \mathrm{~s}$, and it means that the corresponding frame rate is $7500 \mathrm{fps}$. Image size of each figure is $1072 \times 800$ pixels. The LED light source with the power of $300 \mathrm{~W}$ is applied to illuminate the optical view. Cavitation structure inside the impeller shows unsteady characteristics. When the impeller passes the volute tongue, flow structures in the impeller would be significantly affected due to the upstream effect of the volute tongue acting on the impeller. To capture the detailed evolution process of cavitation structures, the region

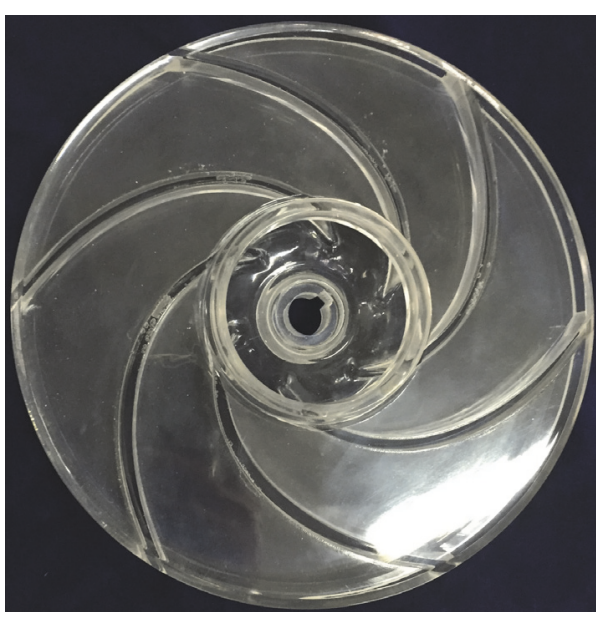

FIGURE 3: The transparent impeller made of Plexiglass for cavitation observation.

including the volute tongue is selected as the view area during visualization measuring, as seen in Figure 4.

\section{Results and Discussions}

3.1. Cavitation Induced Vibration Characteristics. In centrifugal pumps, the net positive suction head is usually defined as in (1). From the head curve of the model pump, the $3 \%$ head drop point is usually considered as the critical net positive suction head $\left(\mathrm{NPSH}_{c}\right)$.

$$
\mathrm{NPSH}=\frac{P_{1}-P_{v}}{\rho g}+\frac{v_{1}^{2}}{2 g},
$$




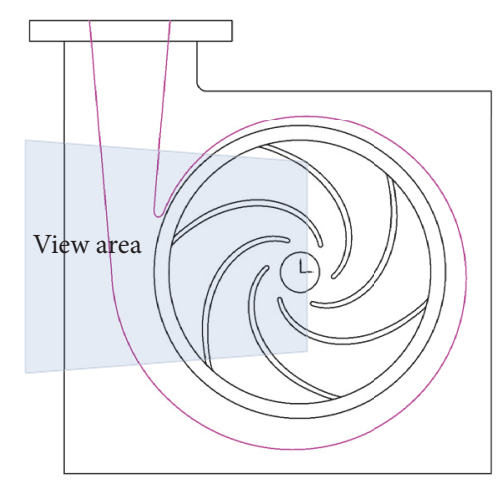

FIGURE 4: View area of optical measurement of the model pump.

where $P_{1}$ is the absolute static pressure at pump inlet, $P_{v}$ is the vapor pressure, and $v_{1}$ is the absolute velocity at pump inlet.

The corresponding cavitation number is defined as in

$$
\sigma=\frac{\mathrm{NPSH}}{H},
$$

where $H$ is the corresponding total delivery head of the pump at nominal flow rate.

Figure 5 first presents cavitation curves of the metal and visualization model pumps at nominal flow rate. $H_{i}$ means the initial pump head at the beginning of cavitation number decreasing. As observed, the curves are nearly identical for the two model pumps. At the beginning stage, with the suction pressure dropping, cavitation bubbles do not develop within the impeller; thus the head curves are not affected by cavitation. As seen, the curves are nearly overlapping at cavitation number $\sigma>0.25$. With the cavitation number decreasing, the head of the metal pump drops faster than the visualization pump. As observed, the head of the metal pump is entirely lower than the visualization pump. At the $3 \%$ head drop point, the critical net positive suction head $\mathrm{NPSH}_{c}$ of the metal pump is $2.0 \mathrm{~m}$; namely, the cavitation number is 0.091 . For the visualization pump, the $\mathrm{NPSH}_{c}$ is $1.97 \mathrm{~m}$, and the cavitation number is 0.0895 . From cavitation curve comparison, it is found that the two pumps almost have the same cavitation curves. So, it is reasonable to establish correlation between cavitation flow and the corresponding induced vibration characteristics using the metal and visualization pumps.

Figure 6 presents cavitation performances at various flow rates of the metal model pump. With the suction pressure decreasing, the pump curves first keep unchanged, then slightly decrease, and finally drop rapidly at the critical point $\mathrm{NPSH}_{c}$. For different flow rates of $0.8 Q_{d}-1.4 Q_{d}$, the $\mathrm{NPSH}_{c}$ is $1.68 \mathrm{~m}, 1.74 \mathrm{~m}, 2.0 \mathrm{~m}, 2.4 \mathrm{~m}, 2.55 \mathrm{~m}, 2.95 \mathrm{~m}$, and $3.3 \mathrm{~m}$, respectively. The corresponding cavitation number is 0.076 , $0.079,0.091,0.109,0.116,0.134$, and 0.150 .

At nominal flow rate, Figure 7 shows vibration spectra of the model pump at $z$-direction of $V 1$ at cavitation and noncavitation conditions, and vibration signals in $0-10 \mathrm{~Hz}$ are filtered. It is observed that cavitation has a significant influence on the vibration spectrum, and almost the whole

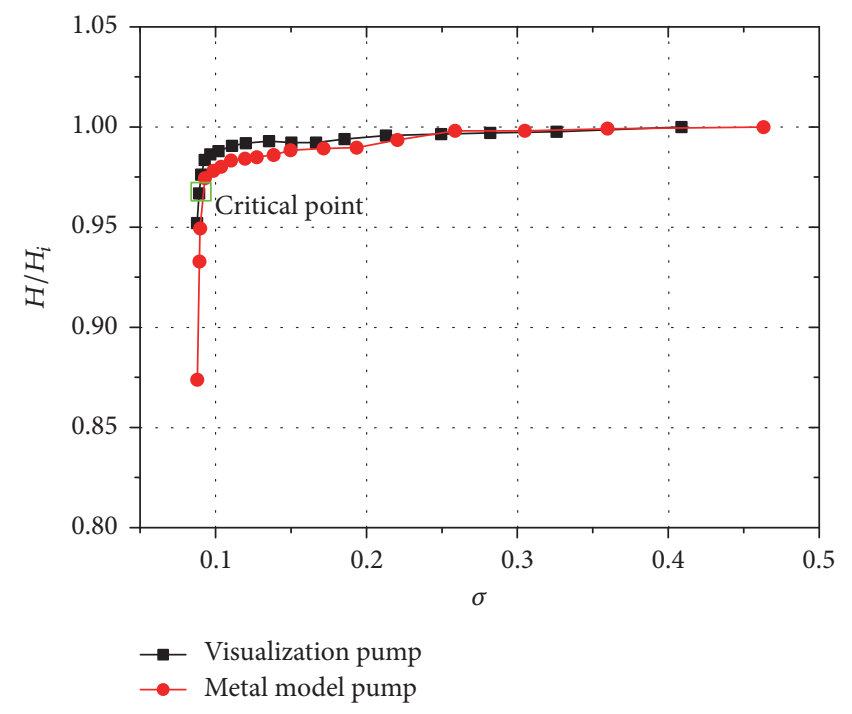

FIGURE 5: Comparison of cavitation curves between the metal and visualization model pumps.

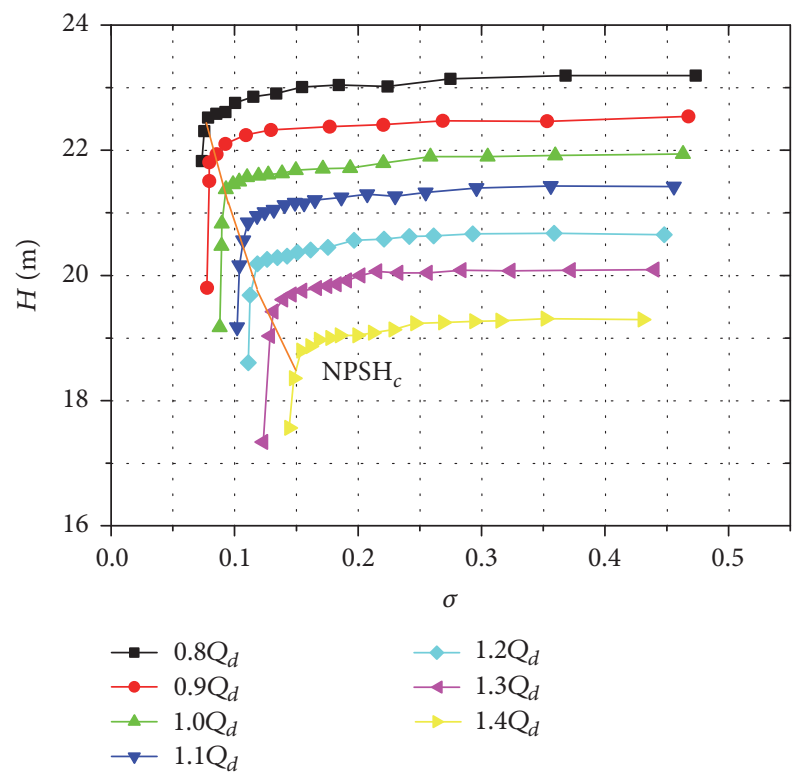

FIGURE 6: Cavitation performances at different flow rates.

frequency band signals are affected. With cavitation number decreasing, high frequency signals in $10000-512000 \mathrm{~Hz}$ band are first affected. It is observed that the amplitudes increase significantly. Especially at full cavitation condition ( $\sigma=0.088$ ), amplitudes of the discrete components increase steeply. Generally, it is accepted that high frequency vibration signals would be excited when cavitation occurs; thus, low frequency signals are rarely investigated in the published studies. Researches have validated that unsteady flow dynamics would induce low frequency signals usually lower than $500 \mathrm{~Hz}$. According to Figure 7, it is found that vibration signals within $10-1000 \mathrm{~Hz}$ are also affected by cavitating flow at small cavitation number, and vibration amplitudes rise compared with that at $\sigma=0.463$. So in the present paper, 

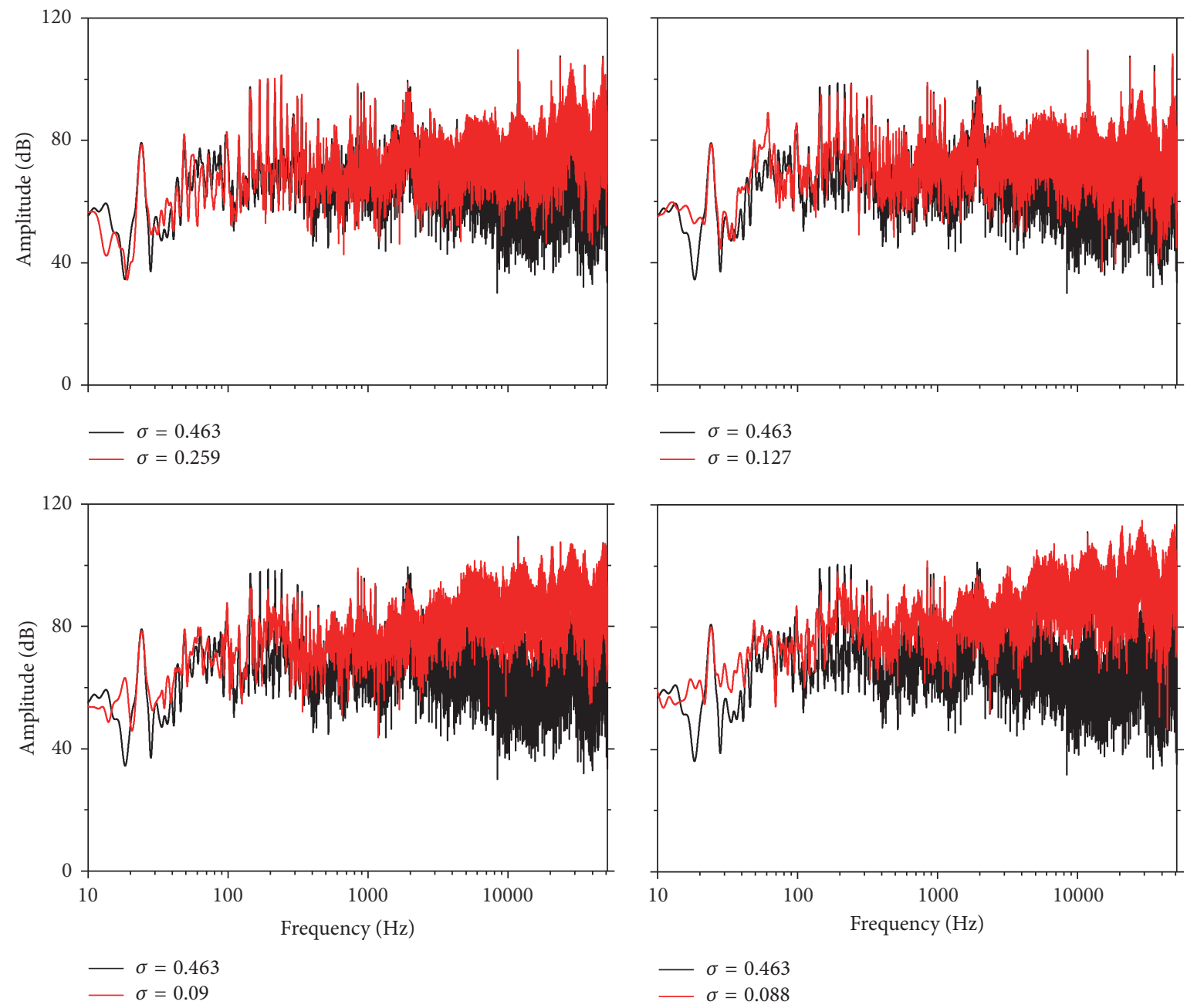

FIGURE 7: Vibration spectra at $z$-direction of $V 1$ with cavitation number decreasing.

vibration signals in full-band of $10-51200 \mathrm{~Hz}$ are analyzed in detail to illustrate the influence of cavitating flow on vibration signals. Meanwhile, vibration spectrum of the model pump is divided into four typical frequency bands for investigation, namely, $10-500 \mathrm{~Hz}, 0.5-10 \mathrm{kHz}, 10-25 \mathrm{kHz}$, and $25-51.2 \mathrm{kHz}$, respectively. Special attention is laid upon cavitating flow induced vibration characteristics in low frequency band of $10-500 \mathrm{~Hz}$.

To evaluate vibration energy versus cavitation number in different frequency bands, root mean square method is applied to deal with components at various frequencies, as defined in

$$
\mathrm{RMS}=\frac{1.63}{2} \sqrt{\frac{1}{2}\left(\frac{1}{2} A_{0}^{2}+\sum_{n=2}^{n-1} A_{n-1}^{2}+\frac{1}{2} A_{n}^{2}\right)},
$$

where $A_{n}$ represents peaks at different frequencies.

Figure 8 presents RMS tendencies along $y$-direction in different frequency bands versus cavitation number when the model pump operates at the nominal flow rate. It is observed that, for high frequency bands of $500-10000 \mathrm{~Hz}$, $10000-25000 \mathrm{~Hz}$, and $25000-51200 \mathrm{~Hz}$, the varying trends are almost identical. As noted, at high cavitation number, RMS values first increase with the suction pressure decreasing. It means that high frequency vibration signals are easily affected by lowering the suction pressure even if cavitation does not occur. The reason may be related to the small bubbles dissolved in the water. With the suction pressure dropping, the dissolved small bubbles would expand. The small bubbles move with the main flow to the high pressure region in the impeller channel; it would explode induced high frequency signals. The emitted high frequency shock wave would result in vibration energy in high frequency band increasing significantly; however vibration signals of low frequency band are nearly unaffected. For RMS values in low frequency band of $10-500 \mathrm{~Hz}$, the varying trends show significant difference. At the beginning stage of lowering the suction pressure, RMS values almost keep unchanged. From Figure 8 it can be seen that when cavitation number is continuously decreasing, RMS values in high frequency band almost remain unchanged showing slight fluctuation at cavitation number $\sigma>0.120$. However for RMS values in $10-500 \mathrm{~Hz}$, the varying trends are completely inconsistent. RMS values increase from cavitation number $\sigma=0.220$ and 

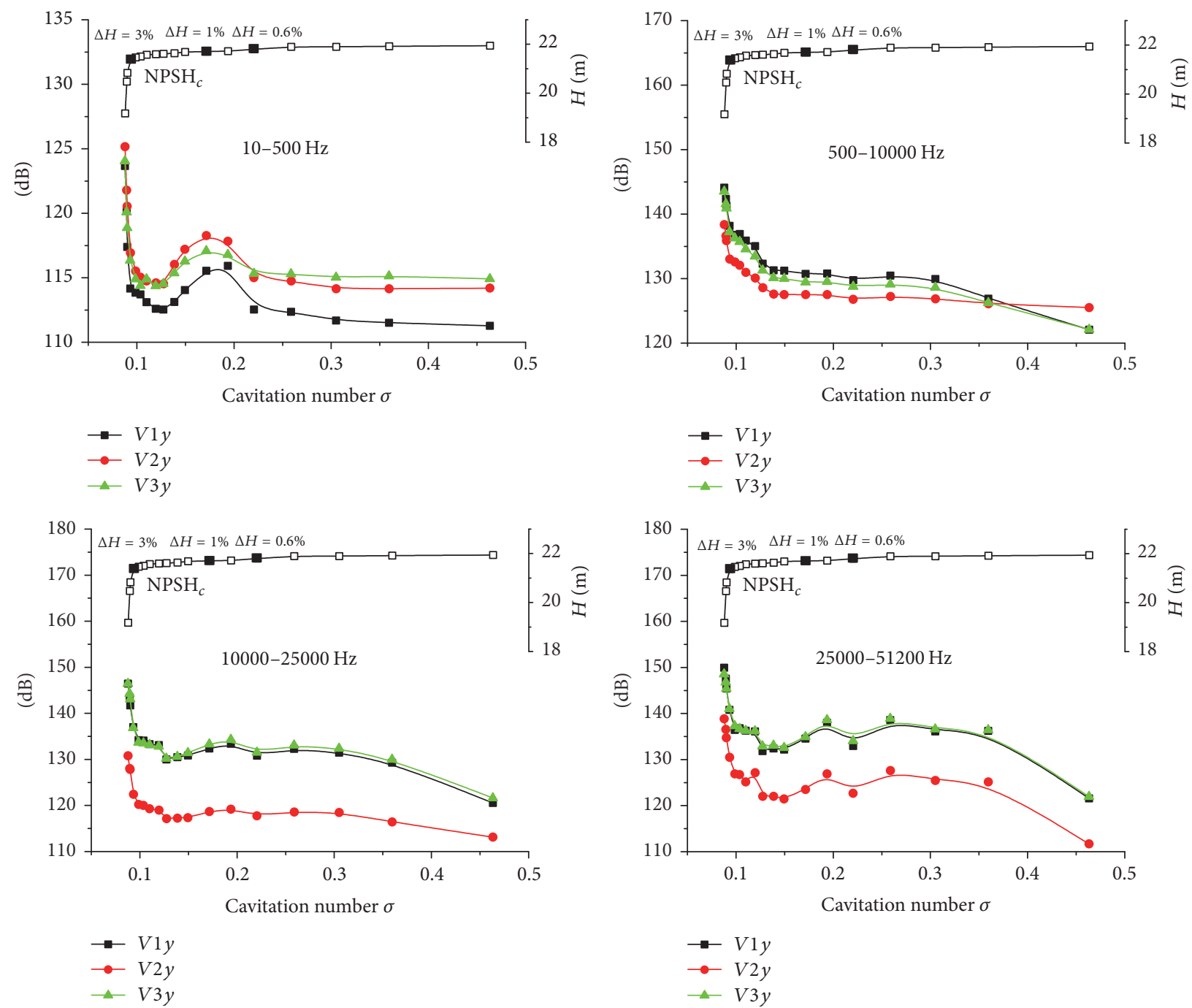

FIGURE 8: RMS values versus cavitation number in different frequency bands along $y$-direction at nominal flow rate.

achieve a local maximum at cavitation number $\sigma=0.171$ with the corresponding $1 \%$ head drop. Then, with cavitation number decreasing to $\sigma=0.120$, vibration energy exhibits a decreasing trend. Finally, with the suction pressure dropping around the critical point, namely, the $3 \%$ head drop point, RMS values increase rapidly for all the concerned frequency bands.

To analyze total vibration energy in three measuring directions, total vibration energy $E$ is used to evaluate vibration energy at different measuring directions, as defined in

$$
E=\sqrt{\frac{\mathrm{RMS}_{x}^{2}+\mathrm{RMS}_{y}^{2}+\mathrm{RMS}_{z}^{2}}{3} .}
$$

Figure 9 presents total vibration energy with the suction pressure decreasing at nominal flow rate. The varying trends in different frequency bands are similar with RMS values. As noted, for vibration energy in $500-10000 \mathrm{~Hz}$, $10000-25000 \mathrm{~Hz}$, and $25000-51200 \mathrm{~Hz}$, the trends of total vibration energy are identical. With the cavitation number decreasing, total vibration energy first increases, then keeps unchanged, and finally rises steeply when cavitation number is lower than the critical point. For total vibration energy in $10-500 \mathrm{~Hz}$ frequency band, vibration energy first remains unchanged, then experiences a local maximum, after that decreases, and finally increases rapidly versus cavitation number dropping lower than the critical point.

The influence of cavitation on low frequency signals is rarely conducted. Attentions are often laid upon the high frequency noise larger than $20 \mathrm{kHz}$ induced by cavitation bubbles as discussed by Mcnulty and Pearsall [16]. From the above analysis, it is concluded that the influence of cavitating flow on vibration energy in different frequency bands is not identical. The varying trend in $10-500 \mathrm{~Hz}$ frequency band is unique. So it is essential to reveal cavitating flow induced vibration characteristics in $10-500 \mathrm{~Hz}$ frequency band at various flow rates. Figure 10 presents RMS values in $10-500 \mathrm{~Hz}$ at flow rate of $0.8 Q_{d}-1.4 Q_{d}$ at $y$-direction of measuring point $V 1$. For various flow rates, the varying trends are similar; namely, RMS values experience a local maximum point 

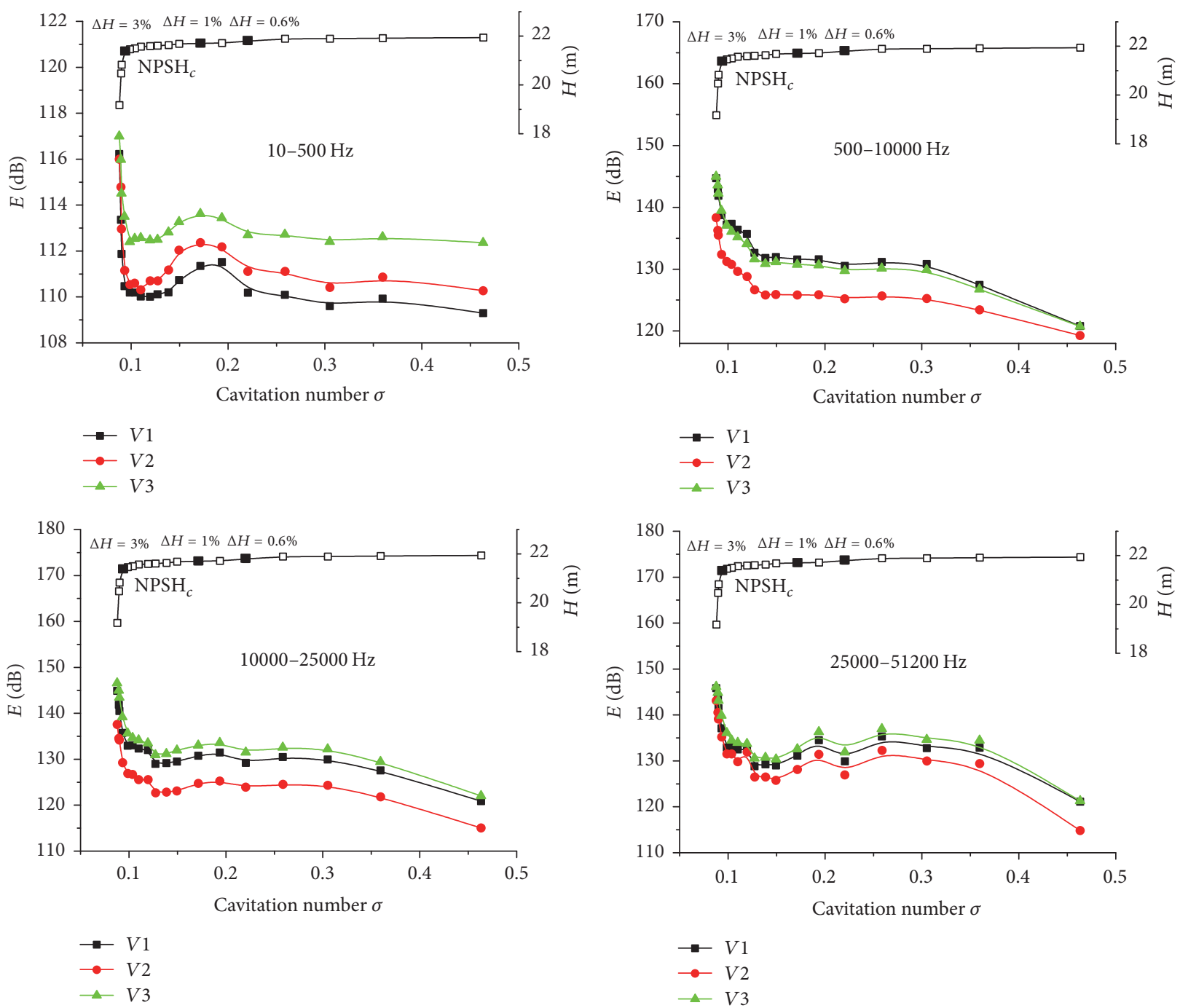

FIGURE 9: Total vibration energy versus cavitation number at the nominal flow rate.

before the 3\% head drop point. Generally, the 3\% head drop point is defined as the critical point, and the corresponding cavitation status within the impeller is considered as full cavitation. However from the view of cavitation induced vibration, it is thought that full cavitation occurs much earlier than the critical point resulting in vibration energy achieving a local maximum point. So in this paper, the square points as presented in Figure 10 are defined as the vibration critical point. For different flow rates, the corresponding head drops at vibration critical point are about $1 \%$ of the starting pump heads. It means that cavitation status at the $1 \%$ head drop point could be considered as vibration full cavitation. Besides, we could also define the inflection points of vibration energy starting to rise as the cavitation inception, namely, the dots in Figure 10. After the inception point, vibration energy in low frequency band is significantly affected by cavitating flow developing in the impeller channels.

Based on the results of Figure 10, we define the cavitation inception point and vibration full cavitation point from the aspect of cavitation induced vibration. Figure 11 presents increments of RMS values from inception point to vibration full cavitation point at various flow conditions. As noted, the increment of the measuring point $V 1$ is much larger than that at points $V 2$ and $V 3$. For points $V 1, V 2$, and $V 3$, the mean increments at different flow rates are $5.2 \mathrm{~dB}, 4.0 \mathrm{~dB}$, and $2.2 \mathrm{~dB}$. To detect the occurrence of cavitation, the increment of RMS value is sufficient in vibration monitoring field, especially for the point $V 1$. So it is concluded that cavitation inside the pump could be detected much earlier than the common $3 \%$ head drop criterion.

3.2. Visualization of Cavitating Flow Structures. From the above analysis, it is validated that the influence of cavitation on vibration characteristics in $10-500 \mathrm{~Hz}$ frequency band is significantly different from that in high frequency bands. It is inferred that the reason is associated with cavitating flow structures within the impeller channels. So we expect to illustrate the internal explanation using visualization of cavitating flow structures. According to the result of RMS values in $10-500 \mathrm{~Hz}$ as shown in Figure 8, we could 


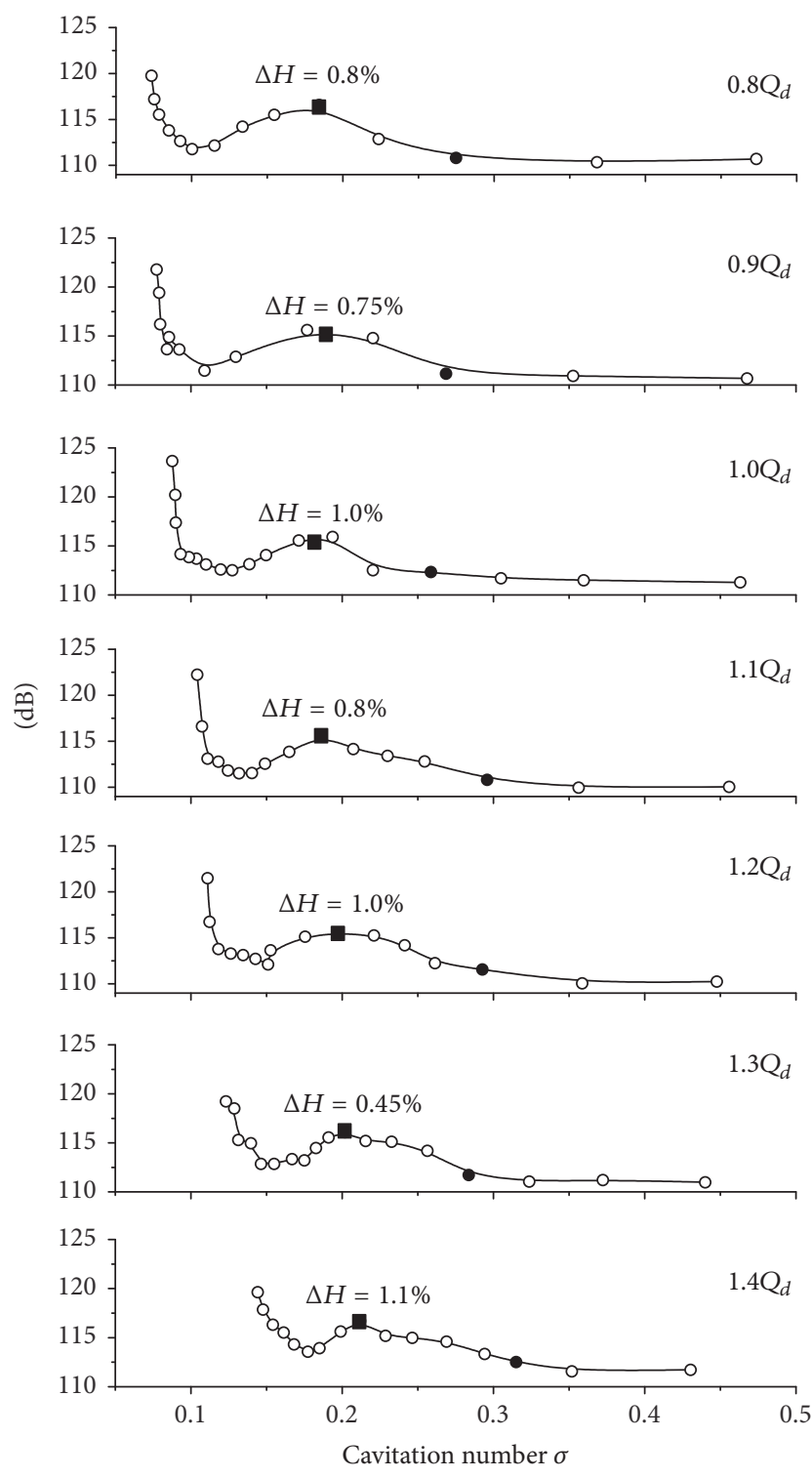

FIGURE 10: RMS values in $10-500 \mathrm{~Hz}$ frequency band versus cavitation number along $y$ direction of $V 1$ under various flow rates.

divide cavitation into four different stages, namely, cavitation inception stage I ( $\sigma=0.463-0.220)$, cavitation developing stage II $(\sigma=0.220-0.171)$, cavitation status transition stage $(\sigma=0.171-0.120)$, and cavitation deterioration stage $(\sigma=$ 0.120-0.088).

At the beginning stage of cavitation, single cavitation bubble or steady sheet cavitation develops in the impeller. Due to partial blade leading edge covered by the suction pipe, it is difficult to capture cavitating flow structures in this stage. So emphasis is laid upon the other three stages. For cavitation developing stage II $(\sigma=0.220-0.171)$, Figure 12 presents cavitation evolution process at cavitation number $\sigma=0.171$. At this stage, with the suction pressure dropping, cavitation region expands showing periodical evolution characteristics. As observed, at $t=t_{0}+30 \Delta t$ moment, partial cavitation cloud starts to separate from the main cavity. With the impeller

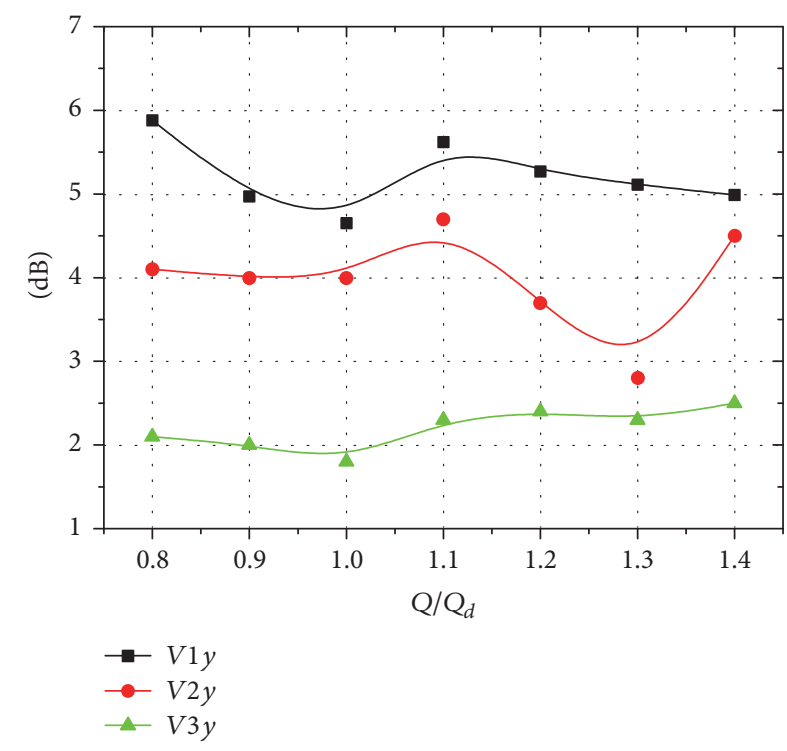

FIGURE 11: Increment of vibration energy from cavitation inception to full cavitation point.

rotating, at $t=t_{0}+30 \Delta t$ and $t=t_{0}+37 \Delta t$ moments, the cavitation cloud experiences detachment and complete separation process. When the cloud is moving towards the impeller outlet with the main flow, cavitation cloud collapses at high pressure region as seen at $t=t_{0}+52 \Delta t$ moment.

From the consecutive figures of cavitating flow structures, it is found that, from cavitation cloud detachment to collapsing, the life cycle of cavitation cloud evolution is $22 \Delta t$, namely, $2.93 \times 10^{-3}$ s. So the shedding frequency of cavitation cloud from the main cavity is $341 \mathrm{~Hz}$ locating in low frequency band $10-500 \mathrm{~Hz}$. From Figure 8 , it is noted that RMS values increase significantly from cavitation $\sigma=$ $0.220-0.171$. It is considered that the reason is associated with the periodically shedding cavitation cloud. Due to the shedding effect of cavitation cloud, vibration energy in low frequency band $10-500 \mathrm{~Hz}$ increases consequently. Meanwhile, the cavitation status at this stage could be defined as cloud cavitation.

With cavitation number lower than 0.171, vibration energy shows decreasing trend from cavitation number $\sigma=$ $0.171-0.120$. Figure 13 presents cavitation developing process at cavitation number $\sigma=0.135$. At this stage, cavitation region is much larger compared with that at $\sigma=0.171$, and some similar evolution processes could also be found at this cavitation number. At $t=t_{0}+11 \Delta t$ moment, cavitation region expands, and partial cavitation cloud tends to separate from the main cavity structure. As observed, with the impeller rotating to $t=t_{0}+26 \Delta t$ moment, cavitation cloud completely sheds from the main cavitation structure. When the detached cavitation cloud is moving to the high pressure region, it would collapse as seen at $t=t_{0}+36 \Delta t$ moment. Finally, the cavitation cloud would disappear in the main flow. From the figures, it is calculated that the life cycle of the cavitation cloud from separating to disappearing is about $38 \Delta t$, namely, 5.05 $\times 10^{-3} \mathrm{~s}$. The corresponding shedding frequency $f_{s}$ is $198 \mathrm{~Hz}$. 

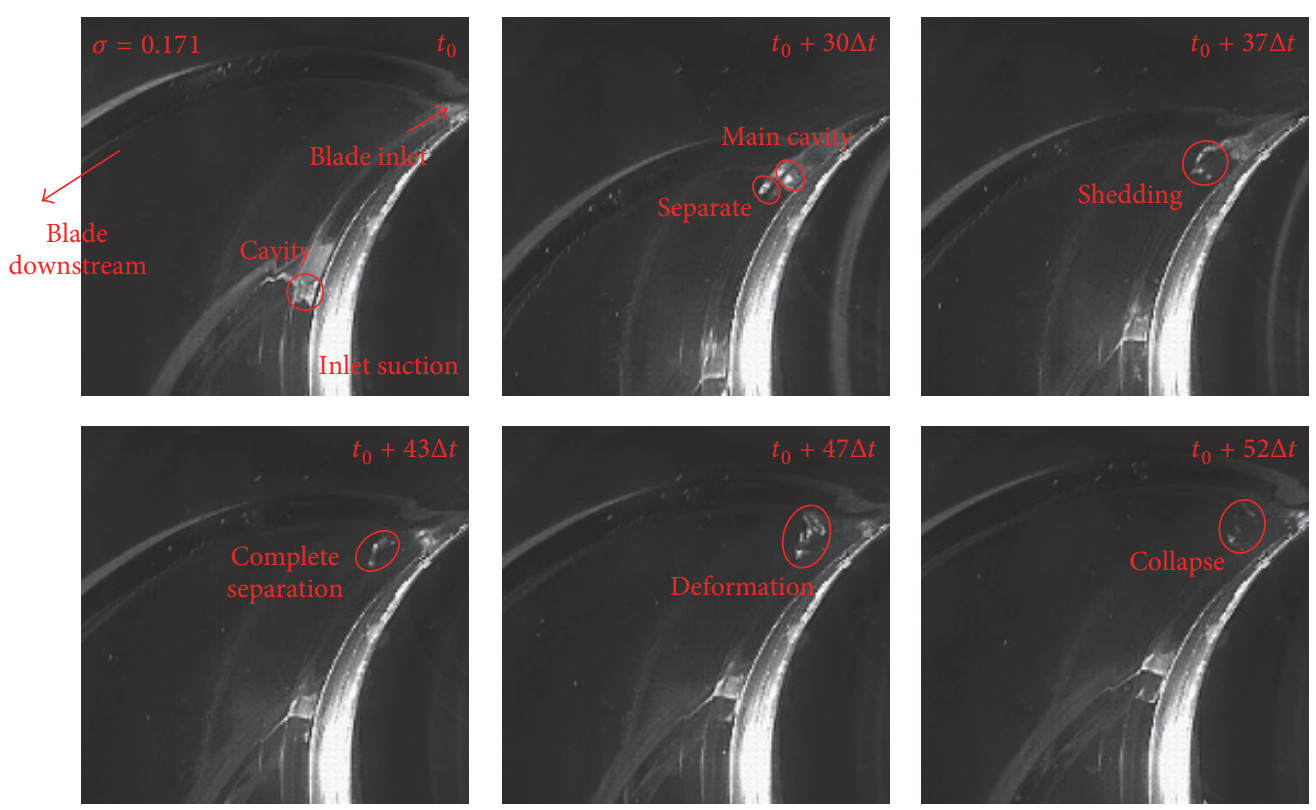

FIGURE 12: Cavitation developing process at cavitation number $\sigma=0.171$.
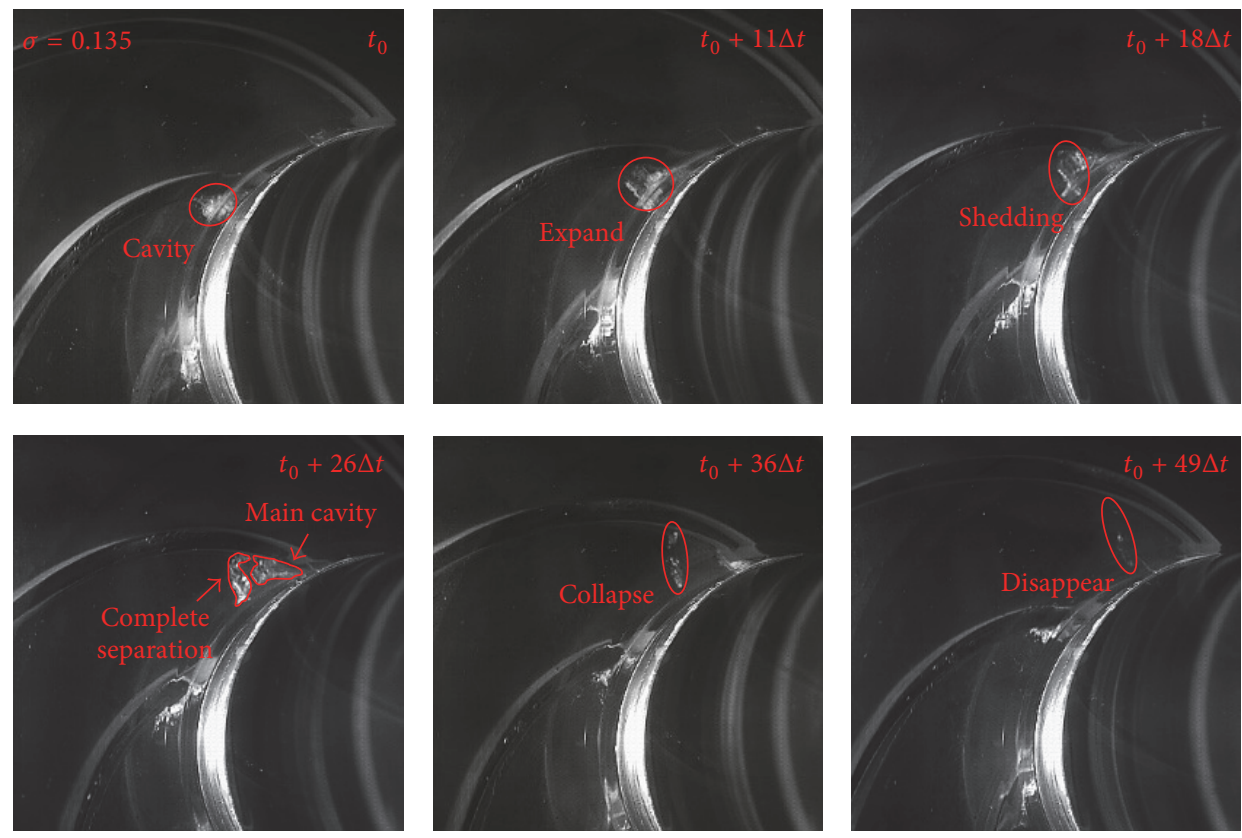

FIGURE 13: Cavitation developing process at cavitation number $\sigma=0.135$.

From the above analysis, it is found that the evolution process of cavitation at $\sigma=0.135$ is similar with that at $\sigma=0.171$. However in this stage, RMS values show opposite varying trend compared with that at $\sigma=0.171$. It means that the emitted energy during cloud collapsing is much lower than that at $\sigma=0.171$. The reason is related to partial compressible characteristics of the cavitation structure. The emitted noise from cavitation bubbles collapsing would be absorbed and attenuated significantly due to the compressible cavitating flow structures. From the published researches, the compressible cavitation structure is usually considered as bubble cavitation. So from cavitation number $\sigma=0.171-0.120$, cavitation status inside the blade channels experiences a transformation process of cloud cavitation to bubble cavitation status.

From the above analysis, it is found that cavitation structures occupy partial blade channels at cavitation number $\sigma>0.120$, but the pump head is almost not affected. With the suction pressure decreasing further to the $3 \%$ head drop point, Figure 14 presents cavitation developing process at 

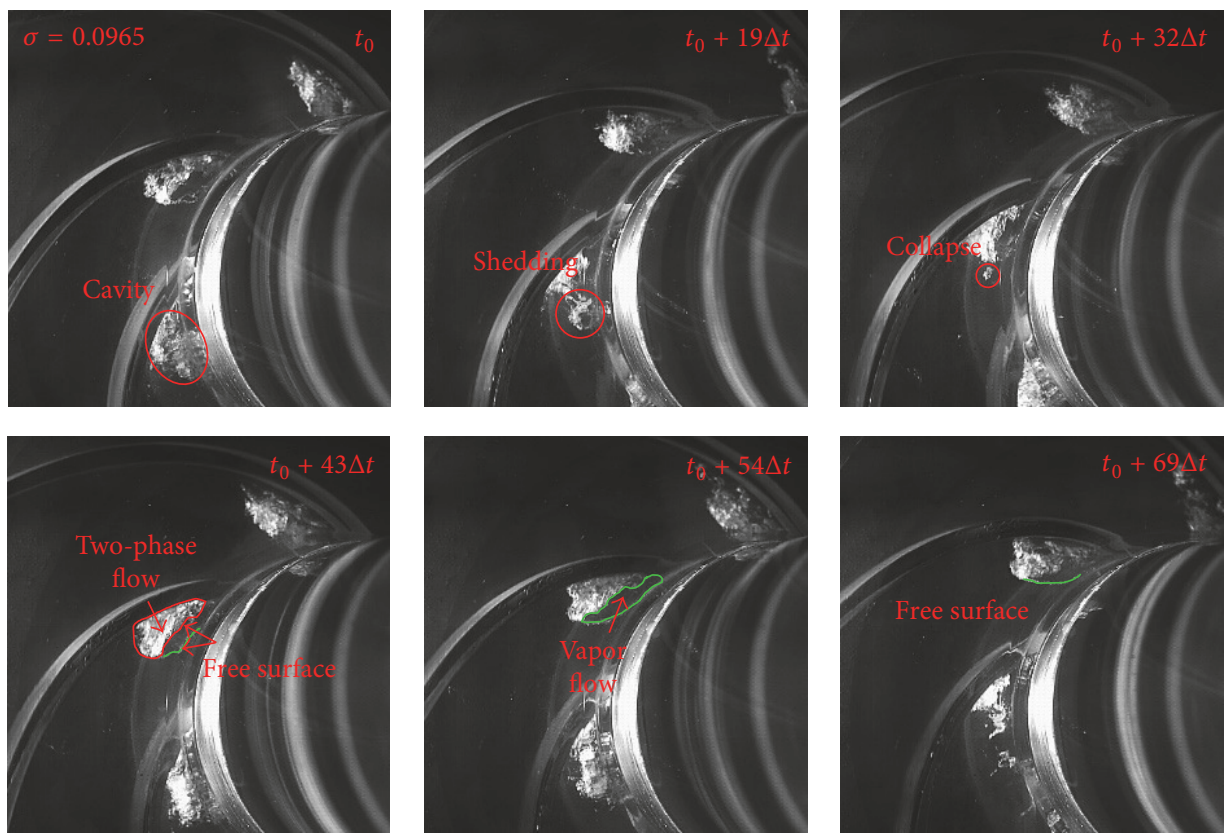

FIGURE 14: Cavitation developing process at cavitation number $\sigma=0.0965$.

cavitation number $\sigma=0.0965$. From the figures, it is noted that, from $t=t_{0}+19 \Delta t$ to $t=t_{0}+32 \Delta t$ moment, partial cavitation cloud sheds from the main cavity and experiences collapsing, disappearing processes. However, significant difference exists compared with the cavitation structures at high cavitation number. As observed at $t=t_{0}+43 \Delta t$ moment, cavitation region could be divided into two separate parts, namely, the two-phase flow region and the vapor flow region. Free surface develops between the two-phase flow and vapor flow; meanwhile, it also exists between vapor flow and the main flow as seen at $t=t_{0}+69 \Delta t$ moment.

From cavitation visualization figures, it is found that free surfaces develop at this stage, namely, cavitation deterioration stage. RMS values at this stage increase rapidly. So it is considered that cavitation structures at this stage are incompressible. Thus the emitted shock wave from cavitation bubbles collapsing could not be attenuated resulting in vibration energy rising steeply around and after the 3\% head drop point. So, from cavitation number $\sigma=0.120-0.088$, cavitation status changes from partially compressible to incompressible characterizing by the significant free surfaces between different phases.

From visualization investigation on cavitating flow structures, it is concluded that the developing process of cavitation flow has a significant influence on vibration characteristics. The varying trends of RMS values in $10-500 \mathrm{~Hz}$ frequency band are associated with the corresponding cavitating flow status within the impeller. In Figure 10, the varying trends at different flow rates are similar, and it means that the evolution processes of cavitating flow at various working conditions are identical as depicted in Figures 12-14.

To investigate the influence of cavitating flow on discrete component at the blade passing frequency $f_{\mathrm{BPF}}$, Figure 15 presents vibration amplitudes at different measuring points versus cavitation number at nominal flow rate. As observed, the varying trends show discrepancy compared with RMS values in $10-500 \mathrm{~Hz}$ frequency band. With cavitation number decreasing, the amplitudes first keep almost unchanged. Even at cavitation number $\sigma=0.171$, vibration energy almost remains constant, and it does not increase significantly. From the starting point to $\sigma=0.171$, vibration amplitudes have an averaged increment of $0.63 \mathrm{~dB}$. Compared with RMS values in $10-500 \mathrm{~Hz}$, at $\sigma=0.171$, RMS values achieve a local maximum, and the mean increment is about $3.5 \mathrm{~dB}$. It means that the periodical shedding cavitation cloud and the collapsing effect have no effect on the amplitude at $f_{\mathrm{BPF}}$. With cavitation number decreasing further, components at $f_{\mathrm{BPF}}$ decrease rapidly, and the amplitudes are even lower than the initial amplitude at the starting point. From the above analysis, it is considered that cavitation structures at this status are partially compressible leading to amplitude at $f_{\mathrm{BPF}}$ decreasing. Then, around the $3 \%$ head drop point, cavitating flow structures are incompressible resulting in amplitudes increasing consequently. The varying trends of amplitudes at $f_{\mathrm{BPF}}$ also validate that cavitating flow structures from cavitation number $\sigma=0.171-0.120$ are compressible.

\section{Conclusions}

In this paper, cavitation flow induced vibration characteristics in a low specific speed centrifugal pump are investigated. Vibration signals are first divided into four frequency bands, and the varying trends of vibration energy versus NPSH are revealed. Meanwhile, cavitating flow structures versus cavitation number are captured using high speed camera to illustrate the influence of cavitation evolution process on vibration energy. The main conclusions obtained in the present paper are as follows. 


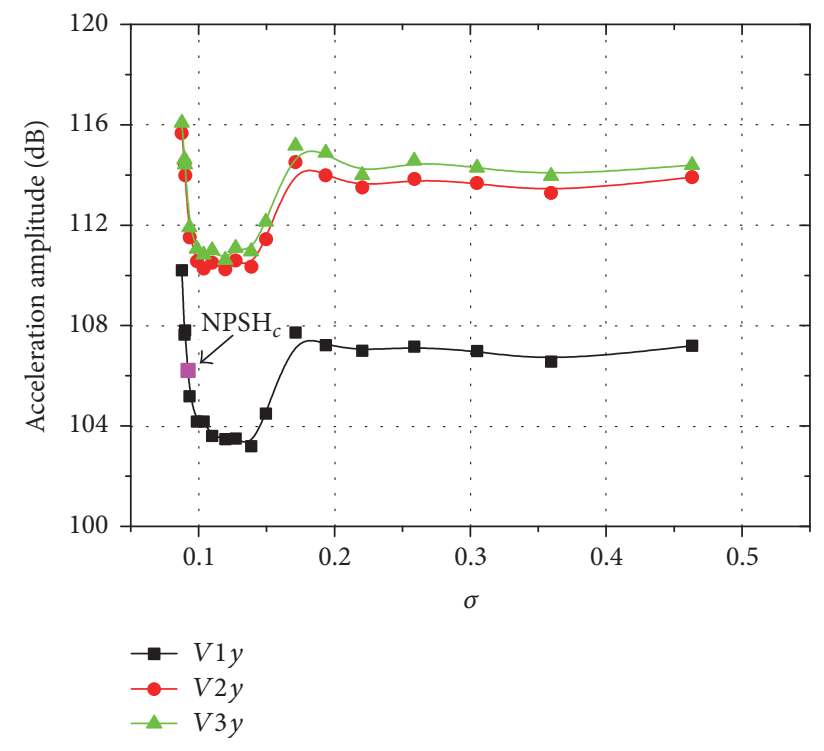

FIGURE 15: Varying trends of amplitudes at $f_{\mathrm{BPF}}$ versus cavitation number at nominal flow rate.

Cavitation in the blade channels has a significant influence on the entire frequency band of vibration signals. Especially for low frequency signals in $10-500 \mathrm{~Hz}$ frequency band, the varying trends are different from that in high frequency band $500-51200 \mathrm{~Hz}$. For RMS values in $10-500 \mathrm{~Hz}$ frequency band, the varying trend experiences four stages. It first keeps almost unchanged, then rises to a local maximum at cavitation number $\sigma=0.171$, after that decreases, and finally increases rapidly around the $3 \%$ head drop point.

The varying trend of RMS value in $10-500 \mathrm{~Hz}$ frequency band is related to cavitating flow structures in the blade channels. From cavitation visualization, cavitation status could be divided into four stages, namely, cavitation inception stage, cavitation developing stage, cavitation status transition stage, and cavitation deterioration stage. At cavitation status transition stage, cavitating flow structures are partially compressible leading to RMS values decreasing significantly.

From vibration energy characteristics, it is found that vibration energy increases much earlier than the $3 \%$ head drop point. From cavitation induced vibration, the local maximum point of RMS value, with the corresponding $1 \%$ head drop, is defined as the vibration full cavitation point. So from cavitation induced vibration, it is concluded that vibration signals can be used to detect cavitation much earlier and more effectively than the conventional 3\% head drop criterion.

\section{Conflicts of Interest}

The authors declare that they have no conflicts of interest.

\section{Acknowledgments}

The authors gratefully acknowledge the financial support of the National Natural Science Foundation of China (51476070,
51576090). The project is supported by the Research Foundation for Advanced Talents of Jiangsu University (16JDG068) and by the Open Research Subject of Key Laboratory of Fluid and Power Machinery (Xihua University), Ministry of Education (szjj2017-096), and funded by the Natural Science Foundation of Jiangsu Province (BK20170547).

\section{References}

[1] C. E. Brennen, Cavitation and Bubble Dynamics, Oxford University Press, New York, NY, USA, 1994.

[2] R. Bachert, B. Stoffel, and M. Dular, "Unsteady cavitation at the tongue of the volute of a centrifugal pump," Journal of Fluids Engineering, Transactions of the ASME, vol. 132, no. 6, pp. 0613011-0613016, 2010.

[3] N. Zhang, M. Yang, B. Gao, and Z. Li, "Vibration characteristics induced by cavitation in a centrifugal pump with slope volute," Shock and Vibration, vol. 2015, Article ID 294980, 10 pages, 2015.

[4] O. Coutier-Delgosha, R. Fortes-Patella, J. L. Reboud, M. Hofmann, and B. Stoffel, "Experimental and numerical studies in a centrifugal pump with two-dimensional curved blades in cavitating condition," Journal of Fluids Engineering, vol. 125, no. 6, pp. 970-978, 2003.

[5] M. Čudina and J. Prezelj, "Detection of cavitation in operation of kinetic pumps. Use of discrete frequency tone in audible spectra," Applied Acoustics, vol. 70, no. 4, pp. 540-546, 2009.

[6] J. Černetič, "The use of noise and vibration signals for detecting cavitation in kinetic pumps," Proceedings of the Institution of Mechanical Engineers, Part C: Journal of Mechanical Engineering Science, vol. 223, no. 7, pp. 1645-1655, 2009.

[7] J. T. Liu, S. H. Liu, Y. L. Wu, L. Jiao, L. Q. Wang, and Y. K. Sun, "Numerical investigation of the hump characteristic of a pumpturbine based on an improved cavitation model," Computers Fluids, vol. 68, pp. 105-111, 2012.

[8] M. Benaouicha, J. A. Astolfil, A. Ducoin, S. Frikha, and O. Coutier-Delgosha, "A numerical study of cavitation induced vibration," in Proceedings of the ASME 2010 Pressure Vessels Piping Division / K-PVP Conference PVP, pp. 18-22, Bellevue, Washington, USA, 2010.

[9] S. Christopher and S. Kumaraswamy, "Identification of critical net positive suction head from noise and vibration in a radial flow pump for different leading edge profiles of the vane," Journal of Fluids Engineering, vol. 135, no. 12, article 121301, 2013.

[10] I. S. Pearsall, "Acoustic detection of cavitation," in Proceedings of the Institution of Mechanical Engineers, Conference Proceedings, vol. 181, pp. 1-8, 1966.

[11] R. Balasubramanian, E. Sabini, and S. Bradshaw, "Influence of impeller leading edge profiles on cavitation and suction performance," in Proceedings of the Second Middle East Turbomachinery Symposium, pp. 17-20, Doha, Qatar, 2012.

[12] M. Čudina and J. Prezelj, "Detection of cavitation in situ operation of kinetic pumps: effect of cavitation on the characteristic discrete frequency component," Applied Acoustics, vol. 70, no. 9, pp. 1175-1182, 2009.

[13] S. Lahdelma and E. K. Juuso, "Vibration Analysis of Cavitation in Kaplan Water Turbines," in Proceedings of the 17th World Congress The International Federation of Automatic Control, pp. 6-11, Seoul, Korea, 2008.

[14] M. Čudina, "Detection of cavitation phenomenon in a centrifugal pump using audible sound," Mechanical Systems and Signal Processing, vol. 17, no. 6, pp. 1335-1347, 2003. 
[15] M. Čudina, "Noise as an indicator of cavitation in a centrifugal pump," Acoustical Physics, vol. 49, no. 4, pp. 463-474, 2003.

[16] P. J. Mcnulty and I. S. Pearsall, "Cavitation inception in pumps," Journal of Fluids Engineering, vol. 104, no. 1, pp. 99-104, 1982.

[17] T. Rus, M. Dular, B. Širok, M. Hočevar, and I. Kern, "An investigation of the relationship between acoustic emission, vibration, noise, and cavitation structures on a Kaplan turbine," Journal of Fluids Engineering, vol. 129, no. 9, pp. 1112-1122, 2007.

[18] N. R. Sakthivel, V. Sugumaran, and S. Babudevasenapati, "Vibration based fault diagnosis of monoblock centrifugal pump using decision tree," Expert Systems with Applications, vol. 37, no. 6, pp. 4040-4049, 2010.

[19] Y. Wang, H. L. Liu, D. X. Liu, J. Wang, and X. F. Wu, "Effects of vane wrap angle on flow induced vibration and noise of centrifugal pumps," Transactions of the Chinese Society of Agricultural Engineering, vol. 29, no. 1, pp. 72-77, 2013. 


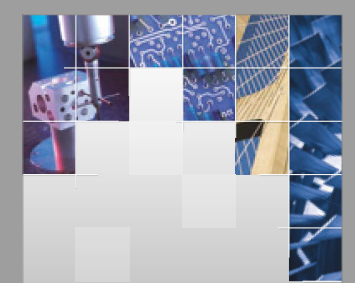

\section{Enfincering}
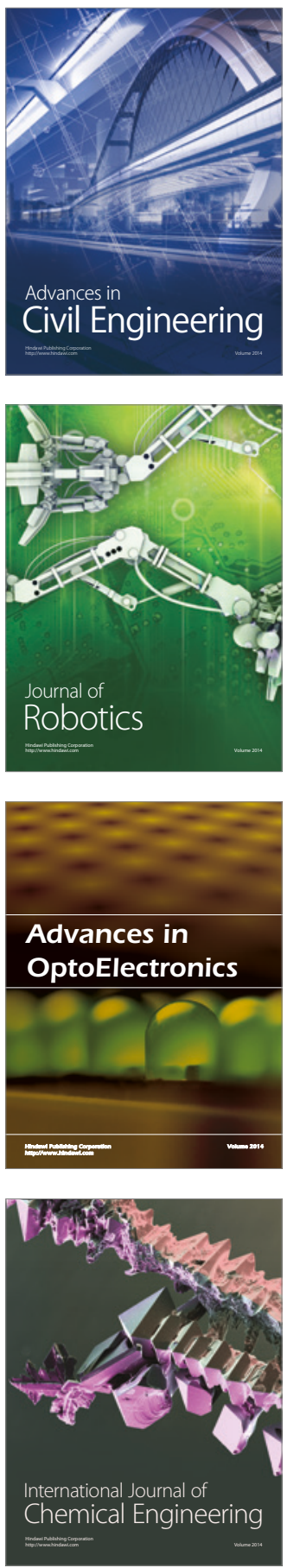

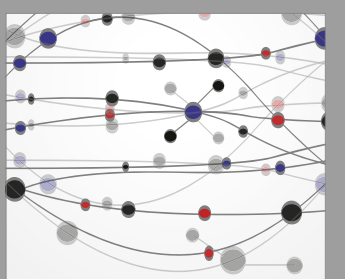

The Scientific World Journal

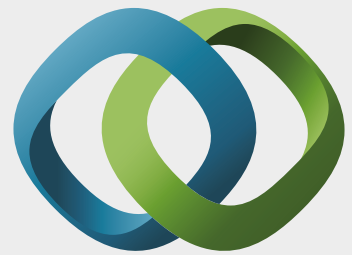

\section{Hindawi}

Submit your manuscripts at

https://www.hindawi.com
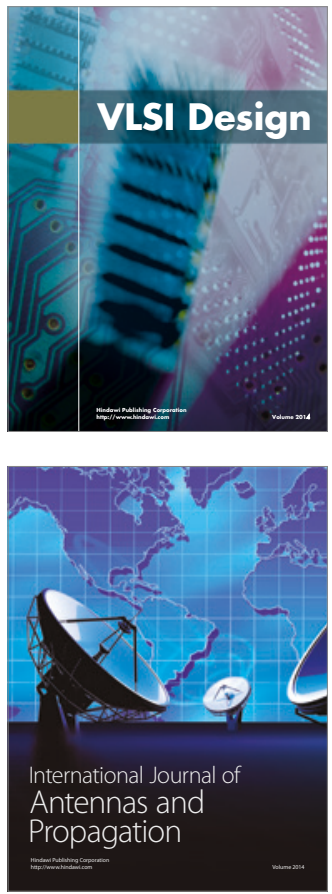

\section{Rotating}

Machinery
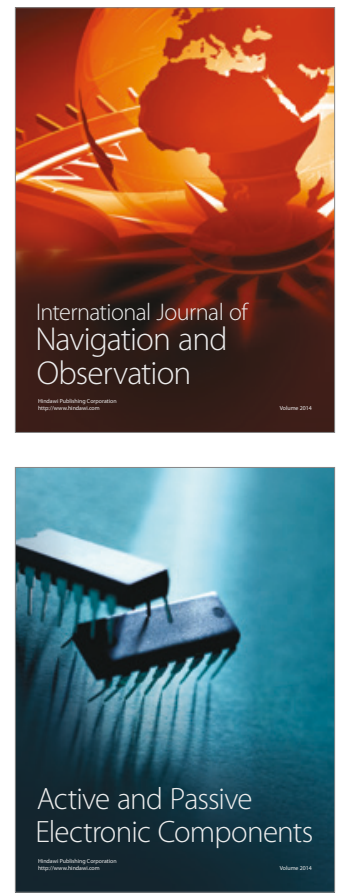
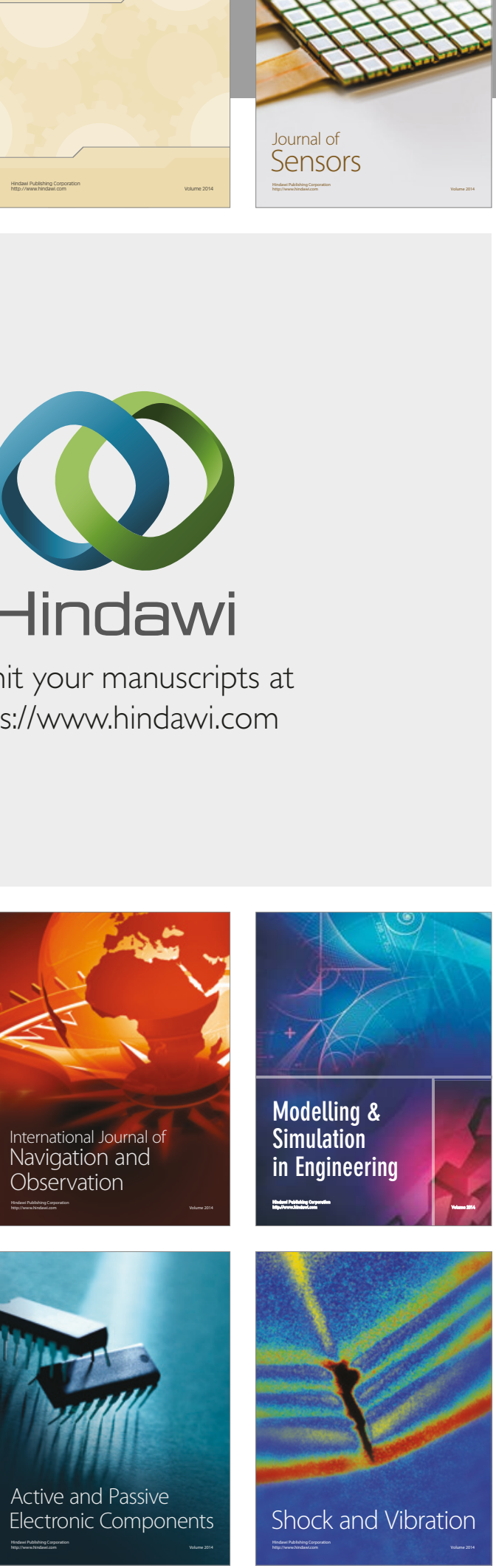
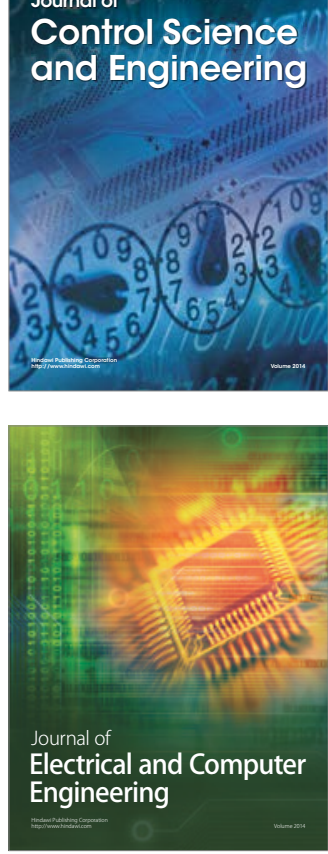

Distributed

Journal of

Control Science

and Engineering
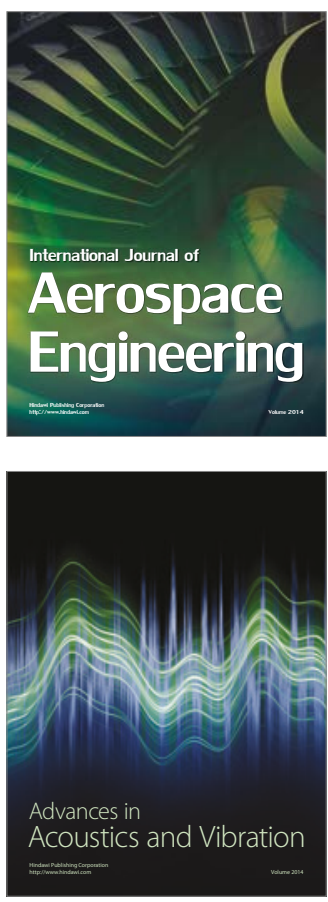

Sensor Networks 